\title{
CROSS-PLY BENDING EQUATION AND APPLICATION FOR COMPRESSED ROD WITH CRACK
}

\author{
I. Pavelko, V. Pavelko
}

\author{
Aviation Institute of Riga Technical University, Lomonosova 1, Riga LV-1019, Latvia \\ E-mail: aviation.institute@rtu.lv; vitaly_pavelko@hotmail.com \\ Received 0707 2004, accepted 13122004
}

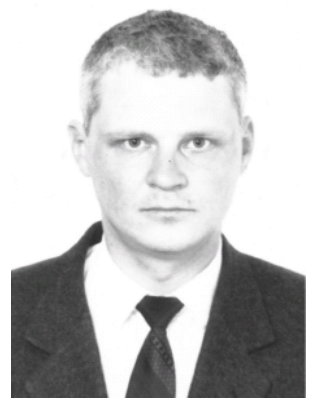

Igor PAVELKO, Dr Sc Eng

Date and place of birth: 1966 in Latvia.

Education: Petersburg Railway Engineering State University (1994), Mechanical Engineer.

Publications: 41 scientific papers, five textbooks and methodological books. Participation in European research project Participation in research projects: Inco-Copernicus Proposal Program N PL964217 (1996 - 1999) and in 6EP project AISHA (2004 - 2006).

Present position: Aviation Institute of Riga Technical University, Assoc Prof of the Aviation Engines Department.

Address: Lomonosova iela 1, LV 1019 Riga, Latvia; Phone +371 7089988, Fax +371 7089990

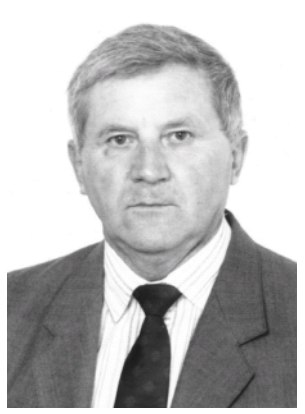

\begin{abstract}
Vitaly PAVELKO, Prof Dr Habil Eng
Date and place of birth: 1941 in Ukraine.

Education: Riga Civil Aviation Engineering Institute, Mechanical Engineer (1963); State University of Latvia, Mathematics (1970). High Doctor Degree in Aviation (1982) in Kiev Civil Aviation Engineering Institute; Doctor Habilitus degree in Engineering (1992).

Publications: More 270 scientific papers, 22 textbooks and methodological books, 14 certificates of inventions, Participation in research projects: European research project Inco-Copernicus Proposal Program N PL964217 (1996 1999), NAS-AURORA (2000-2003), 6FP Project AISHA (2004-2006).

Professional membership: Member of professional Latvian National Committee on Theoretical and Applied Mechanics.

Present position: Professor, Dr Habil Eng., Riga Technical University, Aviation Institute; Head of the Department of the Strength and the Fatigue Durability of Airframe.

Address: Lomonosova iela 1, LV 1019 Riga, Latvia, Phone +371 7089961, Fax +371 7089990.
\end{abstract}

Abstract. The aim of this paper is the analysis of rod cross-ply bending and stability of rod systems in the presence of cracks. The power concept (its theoretical base is Maxwell's theorem about the reciprocity of displacements) and linear fracture mechanics methods for research of mechanical properties of rod systems in the presence of cracks nave been used. The main equation of the rod cross-ply bending and the common solution of this equation were obtained. The expression of the relationship between rod deflection and the disturbing cross-force was obtained. Some transcendental equation allows defining the inferior boundary of critical force of rod with a crack.

Keywords: rod system, cross-ply bending, stability.

\section{Introduction}

In earlier papers, elastic beam systems with cracks in some rods were considered [6, 1, 4, 2, 5, 8]. Some problems of the stress strain state and strength were solved. In particular an attempt to analyze of Euler's problem for axially compressing rod with a crack in some cross-section [5, 6, 2, 7]. The low limit of Euler's critical force was found. In all this research the concept of an elastic multicomponent hinge is used to simulate the influence of a crack on rod compliance. On the basis of this concept, there is the principle of the reciprocity works (Maxwell's theorem). It allows defining a compliance of an equivalent elastic hinge, which influence on integral deformation of a rod coincides accurately with influence of a crack.
$\delta_{\mathrm{j}}=\frac{2}{Q_{j}^{2}} \int_{S}\left[\frac{\left(1-v^{2}\right)}{\mathrm{E}}\left(K_{I}^{2}+\mathrm{K}_{\mathrm{II}}^{2}\right)+\frac{1+v}{E} K_{I I I}^{2}\right] d S,(1)$

where $\delta_{j}$ is the compliance increment (additional generalized displacement) caused by crack influence at an action of the generalized force $\bar{Q}_{j}=1 ; K_{I}, K_{I I}, K_{I I I}$ are stress intensity factors caused by an action of the generalized force $Q_{j} ; E$ is Jung's module and $v$ is Poisson's ratio; $S$ is area of crack surface.

If a rod system is linear, the superposition principle is applicable. Therefore, an influence of several force factors (the axial force, torsion, and bending moments) to the rod deformations may be considered independently from each other in the majority of practical cases of a complex loading. In this situation, the compliance of an equivalent elastic hinge in a direction of the appropriate 
generalized force is constant and does not depend on other force factors at the given area of a crack. However, in some loading cases interference of force factors for the stress strain state of rod is possible and significant. Cases of a buckling and buckling-bending are such. The analogue of the Euler's problem for the compressed rod with a crack was earlier considered. Assuming that influence of axial force for a stress state near a crack front can be neglected, the estimation of critical buckling force was received. It is obvious, that this estimation is the lower border of critical buckling force. Loss of stability at this value of axial force is possible at rather big lateral disturbing. In other words, this critical buckling force we characterize stability possible at the "big disturbing".

In the present paper the problem of a buckling and buckling-bending of the compressed rod with a crack is considered in view of influence of axial force on a stress state near a crack front.

\section{The cross-ply bending problem for a compressed rod with a crack}

The flexible elastic rod of constant cross-section in length $L$ and by hinged fastening on the ends is considered (Figure 1). The axial force $F$ is compressing the rod. In common case there is also bending load $P(z)$. If the rod doesn't have the crack in its some cross-section, it has the critical buckling force $F_{c}$ that can be determined under the known Euler's formula.

If in cross-section with coordinate $L_{1}$ there is a plane crack with the characteristic size $l$, the local compliance of the rod increases in this zone. A rod with an elastic hinge that has equivalent compliance $\delta$ can simulate the total influence of a crack. It is supposed that influence of this hinge on the change of a cross-section turn angle $\Delta \theta=$ $\delta M\left(L_{l}\right)$ for the rod (excluding, perhaps, a small zone near the damaged cross-section) is as such as for the rod with crack.

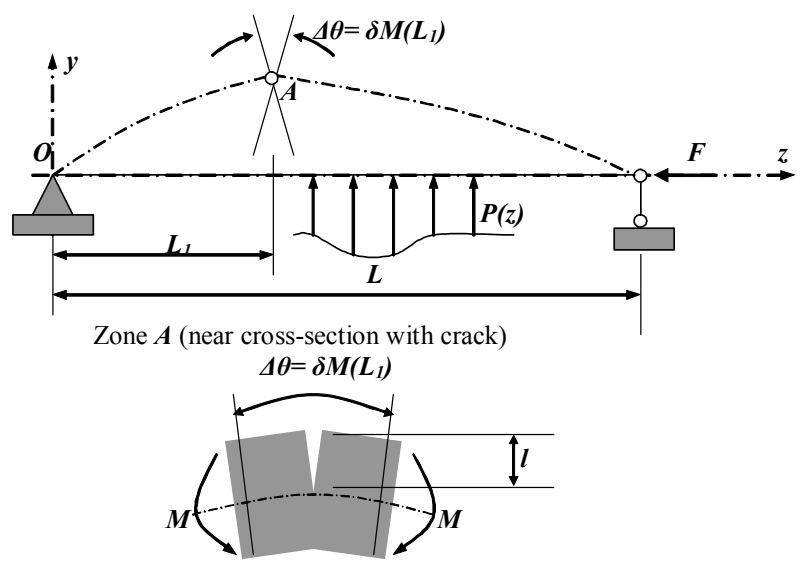

Fig 1. The scheme of elastic rod with a crack

The cross-ply bending differential equation is

$$
\frac{d^{2} v(z)}{d z^{2}}+k^{2} v(z)=\frac{M(z)}{E I},
$$

Where $v(z)$ is the rod deflection, $k^{2}=\frac{F}{E I}$ is a task parameter, $M(z)$ is bending moment, $I$ is the principal moment of inertia of the rod cross-section.

The common solution of the equation (2) looks like

$v(z)= \begin{cases}C_{1} \cos k z+C_{2} \sin k z+v^{*}(z), & \text { if } \mathrm{z} \leq \mathrm{L}_{1} \\ C_{3} \cos k z+C_{4} \sin k z+v^{*}(z), & \text { if } \mathrm{z} \geq \mathrm{L}_{1}\end{cases}$

where $v^{*}(z)$ is the particular solution of equation (2); $C_{i}$ are the constants of integration $(i=1,2,3,4)$ which are determined from boundary conditions

$$
v(0)=0, \quad v\left(L_{1}\right)=0,
$$

and the conditions in cross-section with a crack

$$
v\left(L_{1-}\right)=v\left(L_{1+}\right), \quad \theta\left(L_{1-}\right)=\theta\left(L_{1+}\right)+\delta M\left(L_{1}\right),
$$

Here the particular case of bending load is considered. It is supposed that the concentrated force $P$ acts in the cross-section with a crack. In this case, a bending moment is expressed by formula

$$
M(z)= \begin{cases}-\frac{P\left(L-L_{1}\right) z}{L}, & \text { if } \quad z \leq L_{1} \\ -\frac{P L_{1}(L-z)}{L}, & \text { if } \quad z \geq L_{1}\end{cases}
$$

It is easy to check that the particular solution of equation (2) in this case has the following view:

$$
v^{*}(z)=\left\{\begin{array}{lll}
-\frac{P\left(L-L_{1}\right) z}{k^{2} L E I}, & \text { if } & 0 \leq z \leq L_{1} \\
-\frac{P(L-z) L_{1}}{k^{2} L E I}, & \text { if } & L_{1} \leq z \leq L,
\end{array}\right.
$$

The expressions of the integration constants after some transformations have the following views:

$$
\begin{aligned}
& C_{1}=0, \quad C_{2}=\frac{1}{\sin k L_{1}}\left[v_{0}+\frac{P\left(L-L_{1}\right) L_{1}}{k^{2} L E I}\right], \\
& C_{3}=\frac{\sin k L}{\sin k\left(L-L_{1}\right)}\left[v_{0}+\frac{P\left(L-L_{1}\right) L_{1}}{k^{2} L E I}\right], \\
& C_{4}=\frac{\cos k L}{\sin k\left(L-L_{1}\right)}\left[v_{0}+\frac{P\left(L-L_{1}\right) L_{1}}{k^{2} L E I}\right],
\end{aligned}
$$

where $v_{0}=v\left(L_{l}\right)$ is the rod deflection in cross-section with crack

In a result the common solution of an equation (2) looks like in this case of bending load 
$v(z)= \begin{cases}\frac{\sin k z}{\sin k L_{1}}\left[v_{0}+\frac{P\left(L-L_{1}\right) L_{1}}{k^{2} L E I}\right]-\frac{P\left(L-L_{1}\right) z}{k^{2} L E I}, & \text { if } \quad 0 \leq z \leq L_{1} \\ \frac{\sin k(L-z)}{\sin k\left(L-L_{1}\right)}\left[v_{0}+\frac{P\left(L-L_{1}\right) L_{1}}{k^{2} L E I}\right]-\frac{P(L-z) L_{1}}{k^{2} L E I}, & \text { if } \quad L_{1} \leq z \leq L\end{cases}$

The expressions of the cross-section turn angles are

$\theta(z)=\left\{\begin{array}{l}\frac{\cos k z}{\sin k L_{1}}\left[v_{0} k+\frac{P\left(L-L_{1}\right) L_{1}}{k L E I}\right]-\frac{P\left(L-L_{1}\right)}{k^{2} L E I}, \quad \text { if } \quad 0 \leq z \leq L_{1} \\ -\frac{\cos k(L-z)}{\sin k\left(L-L_{1}\right)}\left[v_{0} k+\frac{P\left(L-L_{1}\right) L_{1}}{k L E I}\right]+\frac{P L_{1}}{k^{2} L E I}, \quad \text { if } \quad L_{1} \leq z \leq L\end{array}\right.$

The rod deflection in cross-section with crack $\boldsymbol{v}_{\boldsymbol{0}}$ is not known. Using (9) and (5), it is possible to express this deflection $v_{\boldsymbol{0}}$ as a function of axial compressing force $F$, normal disturbing force $P$, and the compliance $\delta$.

$\bar{v}_{0}=\bar{P} \bar{L}_{1}\left(1-\bar{L}_{1}\right)\left[\frac{\frac{1}{k L\left(1-\bar{L}_{1}\right) \bar{L}_{1}}}{\frac{1}{\operatorname{tg}\left(k L \bar{L}_{1}\right)}+\frac{1}{\operatorname{tg}\left(k L\left(1-\bar{L}_{1}\right)\right)}-\bar{\delta} k L}-1\right]$

where $\overline{v_{0}}=v_{0} / L, \quad \bar{P}=P / F, \quad \bar{\delta}=\delta / \delta_{0}$ and $\delta_{0}=L / E I$ is the bending compliance of rod when there is not a crack.

\section{The elastic compliance of the equivalent hinge}

It is obvious that critical force $F^{*}$ at the fixed parameters of a problem is determined by the size of a crack and the compliance of an equivalent hinge appropriate to it.

In a considered problem of stress intensity factor (SIF) $K_{I}=K_{I b}-K_{I t}$, where $K_{I b}$ is SIF at a bending, and $K_{I t}$ is SIF at compression. Let crack area be a function one parameter $l$. As a rule then

$$
\begin{aligned}
K_{I b} & =\frac{M}{W} \sqrt{\pi l} \varphi(l / B), \\
K_{I t} & =\frac{F}{A} \sqrt{\pi l} \psi(l / B),
\end{aligned}
$$

where $A$ and $W$ is the area and the axial moment of resistance of a rod cross-section, $B$ is its characteristic size (for example, height), $\varphi(l / B)$ and $\psi(l / B)$ are correction functions on influence of the sizes and form of cross-section.

It is supposed that there is not the shear strain at the crack front. This means that $K_{I I}=K_{I I I}=0$. Then the compliance $\delta$ of an equivalent elastic hinge is

$$
\delta=\frac{2}{M^{2}} \frac{\left(1-v^{2}\right)}{E} \int_{S} K_{I}^{2} d S=\frac{2}{M^{2}} \frac{\left(1-v^{2}\right) t}{E} \int_{0}^{l_{h}}\left(K_{I b}-K_{I t}\right)^{2} d l
$$

where $t$ is cross-section second characteristic size (for example, width); $M$ is an appropriate bending moment in cross-section with an equivalent hinge that depends as from axial force as from bending moment

$$
M=F v_{0}+M_{0},
$$

Here $M_{0}$ is bending moment in the same crosssection at absence of axial force.

Let the rod has rectangular cross-section with the width $t$ and the height $B$. Let too the crack front is straight line that is parallel to horizontal axis of symmetry of cross-section. Then

$$
\bar{\delta}=\frac{\delta}{\delta_{0}}=4 \pi\left(1-v^{2}\right) \bar{B} \int_{0}^{\bar{l}^{*}} x\left\{\varphi(x)-\frac{\bar{B}}{\left[\bar{v}_{0}+\bar{P} \bar{L}_{1}\left(1-\bar{L}_{1}\right)\right] 6} \psi(x)\right\}^{2} d x,
$$

where $\bar{B}=B / L, \quad \bar{L}_{1}=L_{1} / L, \quad \bar{P}=P / F$.

The top limit of integration in the formula (14) $\bar{l}^{*}=\frac{l^{*}}{B}$ is defined by some size $l^{*}$. This size is or the crack actual size $l$, or a root $l_{0}$ of the equation $K_{I b}-K_{I t}=0$ if $0<l_{0}<l$. A crack opens completely in the first case and partly in the second case. It is obvious that $l^{*}=0$ if the crack is not opened at loading.

\section{Condition of closed state of crack}

In the common case, the crack is in closed state when maximal normal stress in cross-section is not positive. It means

$$
\frac{F v_{0}+M_{0}}{F} \frac{A}{W} \leq 1
$$

Using (10) and (15) and accepting $\bar{\delta}=0$, one can define the limit value $P_{0}$ of force $P$. If $P \leq P_{0}$, then the crack is closed to loading.

$$
\begin{aligned}
& \bar{P}_{0}=\frac{P_{0}}{F}= \\
& =2 \bar{i}^{2} \bar{B} \pi \sqrt{\bar{F}}\left(\frac{1}{\operatorname{tg}\left(\pi \sqrt{\bar{F}} \bar{L}_{1}\right)}+\frac{1}{\operatorname{tg}\left(\pi \sqrt{\bar{F}}\left(1-\bar{L}_{1}\right)\right)}\right)
\end{aligned}
$$

$$
\text { where } \bar{F}=\frac{F}{F_{c}}, i=\sqrt{\frac{I}{A}} \text { is the rod cross-section }
$$
radius of inertia, $\overline{\mathrm{i}}=\frac{\mathrm{i}}{\mathrm{B}}$.

It is seen that $\bar{P}_{0}$ is a function of $\bar{F}$ and $\bar{L}_{1}$. This limit force depends also on the form and the relative sizes of a cross-section (multiplier $\frac{\bar{i}^{2}}{\bar{B}}$ ). 


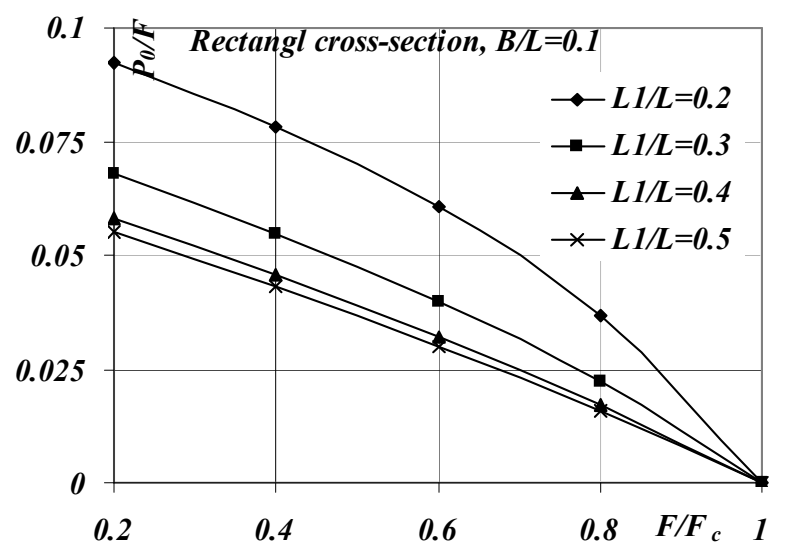

Fig 2. The limit value of bending force as a function of axial force and coordinate of cross-section with crack

In Figure 2, the result of calculation of $\bar{P}_{0}$ for a rod with rectangular cross-section is shown. Obviously that if acted axial force is less than Euler's critical force, then the rod is in a state of equilibrium and the crack is closed. It means the crack doesn't influence to the critical force value at small disturbing actions. This influence will be at the finite actions that not less than some limited value $P_{0}$. At that deflected form of the rod equilibrium may be stable, but at the same time the crack opens. The stable state of equilibrium remains at the disturbing force acting, if it is smaller than some limited value.

\section{Algorithm of the solution of a task}

The algorithm of the solution of the cross-ply bending problem and definition of critical force is following:

1. To check the condition $P \leq P_{0}$ using the formula (16) or (15) in common case.

2. Using the formula (10) to define a deflection $v_{0}$ of a rod at absence of a crack and at the given loading.

3. Under the formula (13) to define bending moment and to check up a condition (15).

4. If this condition (15) is executed, the received solution is final and it is the solution of a classical problem of a cross-ply bending.

5. If a condition (15) is not met, the size $l^{*}$ has be determined.

6. The compliance of the equivalent hinge is calculated as the first approximation. If it is equal or exceeds limiting value under the formula (14), this means that at the given load, rod equilibrium is unstable. In this situation, a calculation repeats from point 1 at reduced load $P$. Otherwise the following point of the algorithm is executed.

7. In the second approximation, the deflection is determined by formula (10) and compared to the first approximation. If the difference is insignificant, calculation comes to an end, but otherwise it repeats from point 2 .

The calculation process will converge, if the configuration is steady. In this case, the solutions give the relationship between the deflection and the total stress intensity factor $K_{I}$. If the rod material is brittle, then the limiting condition of equilibrium on the front of the crack is

$$
K_{I}=K_{I c},
$$

where $K_{I c}$ is the characteristics of the material (the critical stress intensity factor).

\section{The inferior boundary of the critical force of a compressed rod}

From formula (10) it is obvious that even at an infinitesimal bending force, the condition of unlimited increase of a deflection $v_{0}$ comes at some limiting relative compliance $\bar{\delta}^{*}$ of an elastic hinge

$$
\bar{\delta}^{*}=\frac{1}{k L}\left(\frac{1}{\operatorname{tg}\left(k L \bar{L}_{1}\right)}+\frac{1}{\operatorname{tg}\left(k L\left(1-\bar{L}_{1}\right)\right)}\right),
$$

where

$$
k L=\pi \sqrt{\frac{F^{*}}{F_{c}}},
$$

Thus, force $F^{*}$ in the formula (18) represents the critical value of axial force for a rod with a crack.

Earlier it the inferior boundary of the critical force was found using the buckling equation [1]. Here we can define it using (17) and (14), if $v_{0} \rightarrow \infty$. As a result the inferior boundary of the critical force is

$$
F^{*}=k F_{c}=k \frac{\pi^{2} E I}{L^{2}},
$$

where $k$ is the coefficient considering the crack influence.

In Figure 3 the result of this coefficient calculation for rod with rectangle cross-section (the calculation scheme is on Figure 1) is shown.

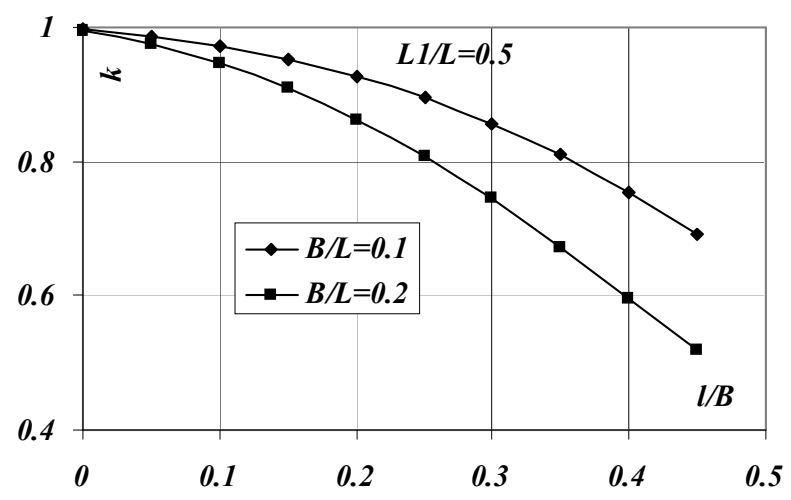

Fig 3. The coefficient crack influence to the critical force of compressed rod with rectangle cross-section

\section{Conclusions}

Use of the power concept of the equivalent elastic hinge for research of mechanical properties of rod 
systems at presence of cracks is an effective means of the analysis of different aspects of airframe strength at presence of the damages. In the present paper this concept has allowed to receive the equation and the common decision of the problem of cross-ply bending and stability of the compressed rod containing a crack in some crosssections of a rod.

It is shown even for the calculated scheme of a rod with hinges on its ends this problem is rather composite. The relation between the rod deflection and the disturbing cross-force is obtained.

It is shown there is some minimal value of the disturbing force before reaching which the crack remains closed. It means in classical introducing the crack does not influence to the value of critical force. Infinitesimal perturbations do not call the deployment of a crack and the change of the rod compatibility. However, if the disturbing force is finite value and more than the mentioned minimum, this effect cannot be neglected. Opening of the crack calls the decrease of stiffness of a rod and downgrade its resistance to the lateral bending. Because of gradual increase of the square of the uncovered part of a crack surface at increase of a disturbing force the compliance of the equivalent elastic hinge increase. Other factor promoting this increase is the gradual increase of the bending effect. The composite relation between the disturbing force, the deflection and the equivalent lowering of the local rigidity of the rod in a zone of crack demands implementation of special composite algorithm of successive approximations for its analysis. However for deriving limiting inferior boundary of critical force some rather simple transcendental equation is obtained. In particular, using this equation in the assumption of final perturbations, it is possible to protect the compressed rods with cracks from the failure and loss of stability.

\section{References}

1. Anderson T.L. Fracture Mechanics: Fundamentals and Applications. - Boca Raton: CRC Press, 1991.$286 \mathrm{p}$.

2. Broek D. Elementary Engineering Fracture Mechanics. - The Netherlands: Martinus Nijhoff, Dordrect, 1986. -4 th ed. -324 p.

3. Elishakoff I. et al. Buckling of Structures: Theory and Experiment. - Elsevier, 1988. - 422 p.

4. Fracture / Edited by H. Libowitz.--New York and London: Academic Press, 1972. - Vol 3.

5. Kanninen M.F., Poplar C.H. Advanced Fracture Mechanics. - Oxford: Oxford University Press, 1985.

6. The Engineering Handbook / Ed. R.C. Dorf //SECTION V: Structures. - Boca Raton: CRC Press LLC, 2000.

7. Pavelko I., Pavelko V. Cross-ply bending and stability of the compressed rod with crack // Scientific bulletin of Moscow State Technical University of Civil Aviation. Section: Aerodynamics and Strength. 2003. - Issue 60. - P.169-178 / In Russian/.

8. Pavelko I., Pavelko V. The valuation of stiffness of rods with cracks. -Warsaw: Informator inst. tech.wojsk lot., 1997. - P. 105-110. 